\title{
Enfoques de aprendizaje en la materia de español en niños con trastornos de aprendizaje
}

\author{
Approaches to learning in the subject of spanish in children with \\ learning disorders
}

\author{
Nadia Vega-Villanuevaa (iD; Gerónimo Mendoza-Meraz ${ }^{(B ;}$; Lilia-Susana Carmona-Garcíac $\mathbb{B}$
}

Recibido: 29-02-2020; Aceptado: 05-05-2020

\section{RESUMEN}

Actualmente, en México, los niños que presentan trastornos de aprendizaje cursan todos los grados escolares junto con los niños regulares. En el estado de Chihuahua el Programa de Servicio Especializado Escolar, a través de un equipo profesional, pretende superar estas problemáticas en los niveles iniciales de la Primaria a los casos más graves, ya que no puede abarcar a toda esta población y, a su vez, proporcionar asistencia específica, a fin de que los niños superen lo más posible las deficiencias que les impide buen desenvolvimiento escolar. Los niños que cursan con trastornos leves deben superar sus problemáticas solos, buscando estrategias de aprendizaje por sí mismos, para consolidar los aprendizajes de cada asignatura. Ante este panorama, el objetivo de la presente investigación fue comparar los Enfoques de Aprendizaje en la materia de español, respecto a los promedios y género de sus alumnos, en los estudiantes con trastornos de aprendizaje que cursan $6^{\circ}$ grado de Primaria en escuelas públicas de la ciudad de Chihuahua, Chih., México. Para llegar a los resultados, se llevó a cabo una investigación no experimental, de campo, comparativo y correlacional, de corte transeccional, realizada en Escuelas Primarias públicas. Se recabaron los datos, aplicando el cuestionario sobre Procesos de Aprendizaje R-LPQ-2F de Biggs, Kember y Leung (2001). Participaron, de forma individual y voluntaria, 227 Alumnos de $6^{\circ}$ grado de Primaria, detectados por el equipo de USAER, con Trastornos de Aprendizaje en 35 escuelas. Resultados. Se observó el empleo de ambos enfoques, en distintos niveles, por parte de los Alumnos participantes. Se encontró predominancia del Enfoque Profundo. Las niñas emplean el Enfoque Profundo más que los niños y no se encontró relación entre los promedios de calificación anual de la materia de Español, con el empleo de ningún tipo de Enfoque de Aprendizaje.

Palabras clave: Enfoques de Aprendizaje; Aprendizaje; Dificultad en el aprendizaje; Estudiante lento; Estudiante de Primaria; Dislexia; Educación especial, Proceso de aprendizaje; Escuela primaria; Psicología de la educación.

aUniversidad Autónoma de Chihuahua, Facultad de Filosofía y Letras, Chihuahua, México. nvega@live.com.mx

bUniversidad Autónoma de Chihuahua, Facultad de Filosofía y Letras, Chihuahua, México. gmendoza@uach.mx

cUniversidad Autónoma de Juárez, Departamento de Ciencias Sociales, Cd. Juárez, México. scarmonag@hotmail.com 


\begin{abstract}
Currently, in Mexico, Children with Learning Disorders attend all grade levels together with Regular Children. In the state of Chihuahua, the Specialized School Service Program, through a professional team, aims to overcome these problems at the initial levels of Primary to the most serious cases, since it cannot cover this entire population and, in turn, provide specific assistance, so that children overcome as much as possible the deficiencies that prevent them from doing well in school. Children who study with Mild Disorders must overcome their problems alone, looking for Learning Strategies by themselves, to consolidate the learning of each subject. Given this panorama, the objective of the present investigation was to compare the Learning Approaches in the subject of Spanish, with respect to the averages and gender of its students, in the Students with Learning Disorders who are in the 6th Grade of Primary in public schools of the Chihuahua City, Chih., Mexico. To reach the results, a non-experimental, comparative and correlational field research was carried out, of a transectional nature, carried out in Public Primary Schools. Data were collected by applying the R-LPQ-2F Learning Process questionnaire from Biggs, Kember and Leung (2001). Two hundred twenty-seven (227) sixth grade students, detected by the USAER team, with Learning Disorders in 35 schools participated individually and voluntarily. Results. The use of both approaches was observed, at different levels, by the participating students. A predominance of Deep Approach was found. Girls use the Deep Approach more than boys, and no relationship was found between the annual grade point averages for Spanish, with the use of any learning approach.
\end{abstract}

Keywords: Learning approaches; Learning; Difficulty in learning; Slow student; Primary student; Dyslexia; Special education, Learning process; Primary school; Psychology of the education.

\title{
INTRODUCCIÓN
}

En los últimos años, ha habido un interés creciente en relación de la educación que recibe la población infantil, como en sus estudios Aguerrondo y Vaillant (2015) mostraron el panorama del contexto educativo deficiente en América Latina. Los reportes realizados por el Laboratorio Latinoamericano de Evaluación de la Calidad (Organización de las Naciones Unidas para la Educación, la Ciencia y la Cultura [UNESCO], 2016) en donde se informa sobre los bajos niveles en el aprendizaje de los Estudiantes y de las necesidades que presentaba la educación en los países latinos (Organización para la Cooperación y el Desarrollo Económico [OCDE], UNESCO y Fondo de las Naciones Unidas para la Infancia [UNICEF], 2016), así como los bajos niveles educativos mostrados por los Estudiantes mexicanos en los resultados del Programa Internacional para la Evaluación del Estudiante (PISA) en el 2018 (OCDE, 2019).

En México, el Modelo Educativo siguió ratificando su adherencia a promover el aprendizaje en el marco de inclusión y equidad; la Secretaría de Educación Pública (SEP) propuso la implementación de innovaciones educativas en donde el alumno consolidara aprendizajes significativos promoviendo en ellos el aprender a aprender (SEP, 2017a). Sin embargo, la evidencia sobre el desempeño siguió mostrando resultados insuficientes en el aprendizaje; muestra de ello, son los resultados del Plan Nacional para la Evaluación de los Aprendizajes (2018), donde se observa que el $49 \%$ de los niños de sexto año lograron un nivel de dominio insuficiente en el área de Lenguaje y Comunicación (Instituto Nacional para la Evaluación de la Educación [INEE], 2019).

El sistema educativo mexicano ha tenido diferentes modificaciones estructurales y de operación, tanto en Educación Básica como en Educación Especial. No obstante, la SEP ha reconocido la necesidad de cambiar las estrategias educativas y modelos de atención a la población con necesidades educativas especiales (SEP, 2013); entre esta población encontramos las niñas y niños que presentan Trastornos de Aprendizaje, cuya atención educativa ha variado en el transcurso de la historia (Sánchez, 2010). 
Vega-Villanueva et al - Enfoques de aprendizaje en la materia de español en niños con trastornos de aprendizaje

En la literatura científica existen diversas denominaciones sobre la conceptualización de los Trastornos de Aprendizaje, dependiendo de la disciplina que lo aborde, desde incapacidad para el aprendizaje, como lo denominó Gearheart (1987); otros como Calderón (1998) le otorgaron el nombre de lesión cerebral mínima y/o disfunción cerebral mínima. García y González (2001) se refirieron a ellos como dificultades de aprendizaje, después, problemas de aprendizaje por Craig y Baucum (2009) y Woolfolk (2010), así como utilizando ambas formas, problemas y dificultades de aprendizaje por Da Fonseca (2013), pero todos ellos describen las mismas dificultades mostradas en los niños tanto para leer, como para escribir, atender y realizar cálculos matemáticos.

El Manual Diagnóstico y Estadístico de los Trastornos Mentales (DSM), versión 5, de la Asociación Americana de Psiquiatría (American Psychiatric Association, APA, 2014), incluye, dentro de los criterios diagnósticos, la identificación de habilidades académicas por debajo de los esperados en la edad cronológica. Dichas especificaciones se determinan mediante el uso de pruebas estandarizadas y el diagnóstico clínico.

El trastorno se presenta en la edad escolar, observándose conductas atípicas en las habilidades académicas como: la lectura se realiza de forma lenta e imprecisa, con dificultad en la comprensión del significado; en la escritura se observan añadiduras, omisiones o sustitución ortográfica, errores gramaticales, dificultades en la expresión escrita, en la forma y el contenido; así como problemas en el dominio del sentido numérico, el cálculo y alteraciones en el razonamiento matemático (APA, 2014), que prevalecen como limitantes para el desempeño escolar, respecto al coeficiente intelectual se presenta en los niveles de la Normalidad a Superior en algunos casos. Estos trastornos excluyen la discapacidad intelectual y/o sensorial, trastornos emocionales o relacionados al medio ambiente (APA, 2014). Clasifica los Trastornos de Aprendizaje en tres dificultades específicas, en la lectura (Dislexia), en la expresión escrita (Disgrafía) y en las matemáticas (Discalculia) (APA, 2014).

Otras problemáticas que pueden presentar estos niños son: alteraciones en el proceso de la atención, algunos de ellos aunado a hiperactividad motora, deficiencias socioemocionales reflejadas en respuestas impulsivas, alteraciones en la percepción visual, auditiva, táctil, espacial, y temporal, dificultades en el proceso de la memoria, alteraciones en el proceso motor, reflejándose en movimientos descoordinados, en torpeza, desbalanceo y desequilibrio y déficits en el proceso de lenguaje (APA, 2014).

En consecuencia, cuenta con lenguaje limitado, déficit cognitivo y metacognitivo, así como alteraciones en la madurez socioemocional, repercutiendo en sus relaciones afectivas con sus pares y medio social (Craig y Baucum, 2009; Da Fonseca, 2013; Gearheart, 1987; Smith Smith, Polloway, Patton y Dowdy, 2004; Woolfolk, 2010). Da Fonseca (2013) sostiene que, aun cuando los niños presentan las alteraciones anteriormente mencionadas, su principal dificultad incide en el aprendizaje en los procesos simbólicos, a pesar de que se procuren circunstancias adecuadas, ya sea en la salud, el ambiente familiar, escolar o social. Algunos de estos niños suelen desarrollar problemas de adaptabilidad social, tanto en el ambiente familiar como el entorno escolar, repercutiendo en su desarrollo del manejo emocional (Smith et ál., 2004) y mostrarse inseguros (Craig y Baucum, 2009). Bryan observó que algunos niños desarrollan sentimientos de sobredependencia y/o desesperanza debido al fracaso académico. Ness y Price, reportaron sentimientos de enojo y frustración; aunado a esto, Wright- Strawderman y Watson encontraron depresión en el 36\% de los casos estudiados (citados en Smith et ál. 2004).

En México, en el 2010, el Instituto Nacional de Estadísticas y Geografía (INEGI) reportó que 9,586 personas presentaban limitación para poner atención o aprender, de las cuales, el 58.13\% era por causas perinatales (INEGI, 2010a). El INEGI reportó en la ciudad de Chihuahua, la existencia de 1,839 personas, entre las edades de 5 a 14 años, con problemas de aprendizaje (INEGI, 2010b). En el 2019, el Departamento de Estadísticas de los Servicios Educativos del Estado de Chihuahua (SEECH) reportó 962 niños y 610 niñas con dificultades severas de aprendizaje, distribuidos desde 
el primer grado hasta el sexto grado de Primaria en diferentes escuelas de cuidad Chihuahua, identificados por los profesores de grupo o el personal de USAER (SEECH, 2019).

Existen diferentes abordajes teóricos respecto a los procesos de consolidación del aprendizaje en el ser humano, que han aportado conocimiento al ámbito educativo y, con ello, enriquecer las herramientas pedagógicas que utilizan los profesores para ayudar a sus Estudiantes a mejorar sus aprendizajes. Dentro de las teorizaciones cuyo objetivo se centra en explicar cómo es que los Estudiantes consolidan su aprendizaje, se encuentra la Teoría de los Enfoques de Aprendizaje. Esta da inicio en diferentes países y en contextos universitarios en la década de los 70 con estudios de Marton y Säljö en Suecia, solos y en equipo (citados en Marton y Säljö, 2005), en el Reino Unido, Entwistle y Ramsden, en equipo entre ellos y con otros investigadores (citados en Entwistle y Ramsden 1983), y en Australia, Biggs, quien retomó las propuestas de Marton y Säljö, así como el constructivismo de Piaget, para dar pauta a la Teoría de Enfoques de Aprendizaje (citado en Biggs, 2010). Creando la línea de investigación Student Approach to Learning (SAL), la investigación sobre los Enfoques de Aprendizaje ha continuado, no solo en Europa sino en Latinoamérica, centrando sus estudios en población universitaria (Soler, Cárdenas, Hernández-Pina y Monroy, 2014).

Biggs enfatiza reflexionar sobre una teoría de aprendizaje que no solo considere la trasmisión de la enseñanza por parte del Docente, sino también el significado del aprendizaje y las actividades de aprendizaje realizadas por el Estudiante. Para Biggs, el aprendizaje del Estudiante depende de los motivos e intenciones, de sus conocimientos previos y de sus estrategias de estudio. De esta forma, el aprendizaje es personal y lo realiza el Estudiante mismo, ya que, aunque en este proceso debe existir una alineación con el contexto de la enseñanza haciendo referencia al Docente y la Institución, es el Alumno quien estructura la información recibida y construye sus aprendizajes a partir de los Enfoques de Aprendizaje que decida utilizar, eligiendo el Estudiante la predominancia hacia el Enfoque Profundo o el Enfoque Superficial (Biggs, 2010).

En el Enfoque Profundo, el objetivo del Estudiante es la comprensión de lo aprendido; el Estudiante muestra interés e importancia a su propio aprendizaje y las actividades académicas que realiza son organizadas, sistemáticas, continuas y reflexivas (Biggs, 2010). En el Enfoque Superficial, el aprendizaje del Estudiante es a nivel memorístico, los contenidos aprendidos son aislados, no realiza análisis de contenidos y ejerce esfuerzos mínimos en las actividades académicas (Biggs, 2010).

Los primeros estudios se realizaron con poblaciones de nivel superior; después, se amplía la investigación en los años 80 a nivel Secundaria se encuentran los estudios de Biggs, Barca y su equipo de trabajo (citados en Soler, 2014) y Mirete, Soro y Maquilón (2015). Considerando esta población estudiantil, Barca (2002) propuso el uso del Sistema Integrado de Evaluación de Atribuciones Causales y Enfoques de Aprendizaje (escala SIACEPA), como una herramienta más al diagnóstico psicopedagógico adecuado para realizarse en entornos formales y pueden estar incluidas personas con dificultades de aprendizaje.

Estudios a nivel Primaria, hasta el momento se tiene referencia de las investigaciones de Maquilón y Hernández (2011) y Maquilón, Sánchez y Cuestas (2016) en población infantil Española; ellos encontraron el uso mayor de un enfoque de Alto Rendimiento (variable agregada a su estudio), seguido del Enfoque Profundo; en México, García (2016) observó predominancia del Enfoque Profundo sobre el Enfoque Superficial en una población de Alumnos de nivel Primaria.

Ante tales hallazgos se formulan las siguientes preguntas de investigación, ¿Predomina algún tipo de Enfoque en la materia de Español en Alumnos que presentan Trastorno de Aprendizaje? ¿Existe relación entre el promedio obtenido en la materia de Español respecto al tipo de Enfoque que emplea el Alumno con Trastorno de Aprendizaje? ¿Los niños emplean, en diferente medida, tanto el Enfoque Profundo como el Superficial que las niñas? Esta falta de información en poblaciones mexicanas, especialmente en niños y más aún, en niños con Trastornos de Aprendizaje, subraya la necesidad de estudiar con más detalle el empleo de los Enfoques de Aprendizaje. 
Vega-Villanueva et al - Enfoques de aprendizaje en la materia de español en niños con trastornos de aprendizaje

De tal manera que la presente investigación tiene como objetivo comparar los Enfoques de Aprendizaje en la materia de Español, respecto a los promedios y género de sus Alumnos, en los Estudiantes con Trastornos de Aprendizaje que cursan $6^{\circ}$ grado de Primaria, en escuelas públicas de la ciudad de Chihuahua, Chih., México.

\section{METODO}

Variables de Estudio. 1. Enfoques de Aprendizaje, refiriéndose a las actividades, motivos y estrategias realizadas por el Estudiante, centradas en la adquisición del aprendizaje, tomado del concepto de Biggs, (2010) en este caso hacia la materia de Español. Categorizado en: Enfoque Profundo. Donde el Estudiante expresa interés y es participativo de su propio aprendizaje, organiza y sistematiza actividades a fin de comprender, realizar análisis y da significado de sus aprendizajes con entusiasmo, su interés es aprender de manera profunda. Y se observó a través de las respuestas emitidas por escrito en el instrumento aplicado. Enfoque Superficial, cuyas características obedecen a que el Estudiante no está interesado en aprender de forma profunda, realiza actividades que requieren un mínimo nivel cognitivo, su aprendizaje solo se queda a nivel memorístico, no realiza análisis profundos de los contenidos, se centra en datos concretos, los contenidos que aprende son aislados, solo le interesa aprobar; por lo tanto, sus esfuerzos son mínimos y se observó a través de las respuestas emitidas por escrito en el instrumento aplicado. 2. Promedio anual de la materia de Español, obtenido del total de calificaciones de las evaluaciones realizadas en todo el año y divididas entre el número de ellas, realizado por cada Maestro y proporcionado a la investigadora responsable por la SEECH.

Hipótesis. $\mathrm{H}_{\mathrm{o} 1}$ No existe predominancia de algún tipo de enfoque en los Alumnos de Trastornos de Aprendizaje respecto a la materia de Español. $\mathrm{H}_{02}$. No existe una relación entre el promedio obtenido en Español respecto al empleo de un tipo de Enfoque de Aprendizaje. $\mathrm{H}_{\text {o3. }}$ Los niños emplean en menor medida el Enfoque Profundo que las niñas.

Diseño de Investigación. Investigación cuantitativa, no experimental, de campo realizada en planteles educativos públicos de nivel Primaria de ciudad Chihuahua, Chih., comparativo, correlacional y de corte transeccional.

Población y Muestra. Los participantes fueron 227 Alumnos, entre 10 a 12 años de edad cronológica, de ambos sexos, que al momento del estudio cursaban $6^{\circ}$ año de una Primaria en una escuela pública y cuya característica de inclusión en el estudio fue que presentaran algún Trastorno de Aprendizaje, ya fuere dislexia, disgrafía o discalculia, identificados previamente por el Docente del grupo o por un especialista del equipo de USAER con base en los criterios establecidos por el APA (2014), para identificar las dificultades existentes en los Alumnos de forma uniforme. La participación de los Estudiantes fue voluntaria, se contó con el consentimiento confirmado de los padres de familia en su calidad de apoderados legales de ellos, y con los permisos de las instancias educativas para la realización del estudio.

Instrumento. Para el levantamiento de datos se utilizó el cuestionario sobre Procesos de Aprendizaje R-LPQ-2F de Biggs, Kember y Leung (2001); mide el empleo de los Enfoques de Aprendizaje de los Alumnos, conformado por 18 ítems; diseñado como escala tipo Likert de cinco opciones de respuesta, que van de 1 a 5, donde el 1 es Nulo Empleo y 5 es Continuo. El instrumento está dividido en dos factores: Enfoque Profundo y Enfoque Superficial, como se especificó en las variables; el instrumento ha sido utilizado en poblaciones diversas, tanto en países europeos como latinos, en mayor medida en Estudiantes universitarios que en los demás niveles educativos y en menor escala en niños a nivel Primaria, con un ALFA de .81 en el Enfoque Profundo y en Enfoque Superficial, ALFA de .70. 
Análisis Estadístico. Con la finalidad de analizar los datos obtenidos se utilizaron estadísticas descriptivas, para observar la distribución de frecuencias y medidas de tendencia central a fin de describir el comportamiento de las variables de estudio, y al comparar los grupos se utilizaron la prueba t de Student, así el ANOVA, para lo cual se empleó el programa computacional Stadistical Parchage for Social Sciences (SPSS, 22).

\section{RESULTADOS}

La población de estudio estuvo conformada por 158 varones, que corresponde al $70 \%$ de los Alumnos y 69 mujeres, siendo el $30 \%$ de la población. La edad de los niños se encontró distribuida por niños de 10 años en un 6\%, de 11 años corresponden al 76\% y los niños de 12 años al $19 \%$ de los Alumnos.

Respecto a las calificaciones, el promedio en Español que obtuvieron los Alumnos en general fue de 7.8, con una $s d=1.04$, en un rango de 6.0 a 10.0, donde la calificación más común fue de 8.0. El $59 \%$ de los Estudiantes presentaron calificaciones entre 6.0 y 8.0 y el $41 \%$ sostuvo promedios entre 8.1 y 10.0. En cuanto a las calificaciones anuales, en relación al género de los Alumnos, las niñas obtuvieron un promedio de 8.2 , con una $\mathrm{sd}=.98$, en un rango de 6.0 a 10.0 y la calificación promedio que más se repitió fue 7.6 ; el $48 \%$ de las niñas mostraron promedios entre 6.0 y 8.0 , mientras que el $52 \%$ se mantuvo entre el 8.1 y el 10.0 . En cuanto a los varones, el promedio obtenido ascendió a 7.8 y una $\mathrm{sd}=1.04$, con un rango entre 6.0 a 10.0 de calificaciones promedio, siendo 7.0 la calificación que más se presentó; el 63\% de los varones obtuvieron calificaciones entre 6.0 y 8.0 y el $37 \%$ de 8.1 y 10.0 .

Enfoque de Aprendizaje Profundo. En la Figura 1 se observa que los Alumnos con Trastornos de Aprendizaje presentan en mayor porcentaje una frecuencia moderada (51\%) en el empleo del Enfoque Profundo, mostrando porcentajes más cargados hacia los niveles más altos.

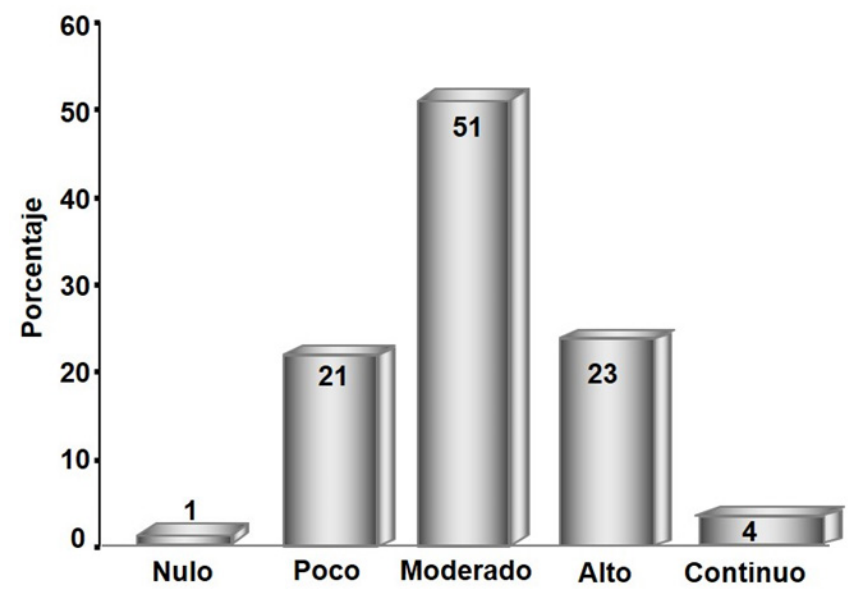

Figura 1. Distribución de Frecuencias del Enfoque Profundo.

Enfoque de Aprendizaje Superficial. La Figura 2 muestra que los Alumnos que cursan con Trastornos de Aprendizaje presentan un mayor porcentaje (65\%) en los niveles de frecuencia entre Nulo y Poco Empleo del Enfoque Superficial. 


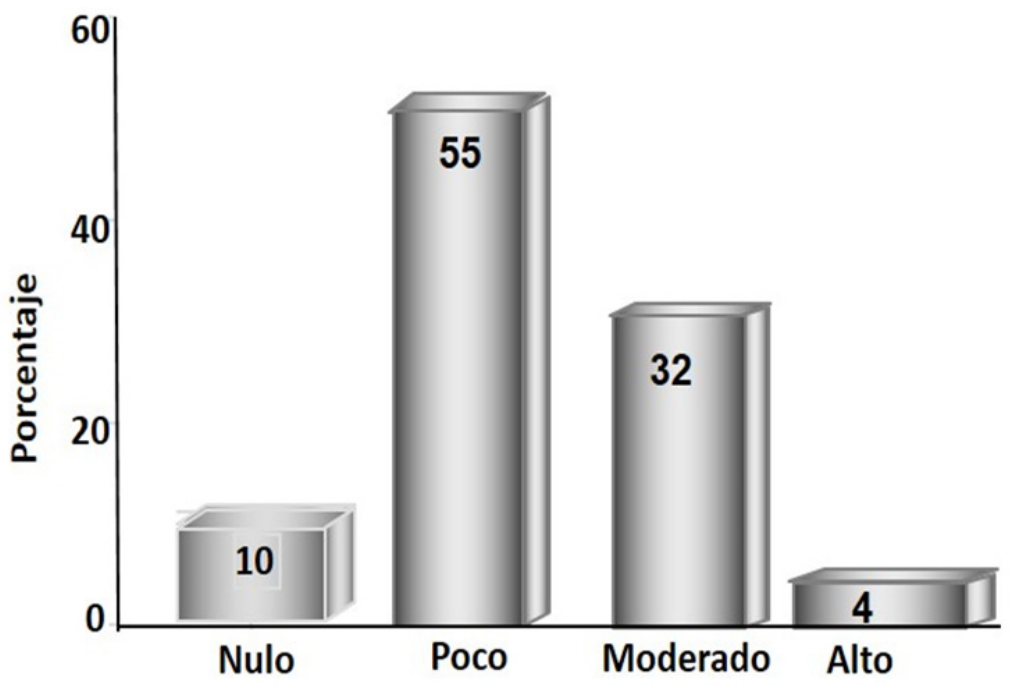

Figura 2. Distribución de frecuencias del Enfoque Superficial.

Predominancia de los Enfoques de Aprendizaje, Profundo y Superficial. Al comparar el empleo del Enfoque Profundo con el Enfoque Supercial de parte de los Alumnos, se encontró una dominancia del Enfoque Profundo mostrada en la prueba $t$ de Student con un valor de $\mathrm{t}(226)=11.34, \mathrm{p}=.00, d=1.15$, respecto al Enfoque Superficial. Las medidas de tendencia central en el Enfoque Profundo son $(M=30.96$, $s d=7.46, n=227)$, en tanto que en el Enfoque Superficial son $(M=22.85, s d=6.67, n=227)$.

Predominancia de los Enfoques de Aprendizaje Respecto al Género. Enfoque Profundo. Se encontró que la niñas emplean en mayor medida que los niños el Enfoque Profundo. Los resultados de la prueba $\mathrm{t}$ de Student arrojaron un valor de $\mathrm{t}(126.8)=2.35, \mathrm{p}=.02, d=0.34$, afirmando que existe una diferencia estadística significativa a favor de las niñas. Las medidas de tendencia central en el Enfoque Profundo de las niñas son $(M=32.72$, sd=7.50, $n=69)$, y en los niños $(M=30.20$, sd=7.32, $n=158)$. Enfoque Superficial. Los resultados de la prueba t mostraron una diferencia estadísticamente significativa mayor en los niños con un valor de $\mathrm{t}(125.7)=2.019$, $\mathrm{p}=.04, d=0.30$ respecto a las niñas. Las medidas de tendencia central en los niños son: $(M=23.45$, $\mathrm{sd}=6.55, \mathrm{n}=158)$, mientras que en las niñas son: $(M=21.49, \mathrm{sd}=6.78, \mathrm{n}=69)$.

Relación entre los Enfoques de Aprendizaje y Calificaciones. Como se muestra en la Tabla 1 , en la correlación de Pearson no se apreciaron relaciones que resaltar entre los promedios de las calificaciones anuales de la materia de Español, obtenidas por los Alumnos de $6^{\circ}$ grado, que cursan con Trastornos de Aprendizaje y el empleo del Enfoque Profundo; en cuanto al Enfoque Superficial, se encuentra una relación negativa muy baja pero significativa con el promedio de calificación anual.

Tabla 1. Correlación entre la Calificación Anual respecto a los Enfoques Profundo y Superficial.

\begin{tabular}{clccc}
\hline $\mathbf{N}=\mathbf{2 2 7}$ & & Calificación & Enfoque Profundo & Enfoque Superficial \\
\hline Calificación & $r$ Pearson & 1 & .082 & $-.194^{* *}$ \\
& $p$ bilateral & & .217 & .003 \\
Enfoque Profundo & $r$ Pearson & .082 & 1 & $-.156^{*}$ \\
& $p$ bilateral & .217 & & .019 \\
\multirow{2}{*}{ Enfoque Superficial } & $r$ Pearson & $-.194 * *$ & $-.156^{*}$ & 1 \\
& $p$ bilateral & .003 & .019 & \\
\hline
\end{tabular}

** La correlación es significativa al nivel del 0.01 (bilateral);

* La correlación es significativa al nivel del 0.05 (bilateral) 


\section{DISCUSION}

Los resultados de esta investigación se centran en el empleo de los Enfoques de Aprendizaje de los Alumnos de $6^{\circ}$ grado de nivel escolar básico que cursan con Trastornos de Aprendizaje en la ciudad de Chihuahua, Chih., México, abarcando los 4 puntos cardinales de la localidad, cuidando de esta forma que la muestra fuere representativa de la población citada en las escuelas Primarias públicas de la ciudad. Investigación que resulta relevante por varios aspectos: uno, relacionado con el enriquecimiento que aporta al tema de estudio, dada la poca atención brindada por parte de los estudiosos de los fenómenos que atañen al contexto de la educación en la República Mexicana y específicamente a nivel Primaria; y, otro, por la población estudiada, teniendo en cuenta que a los Alumnos que cursan con Trastornos de Aprendizaje en nivel bajo y regular, no son motivo de atención por los especialistas en las escuelas Primarias. Por lo tanto, ellos buscan solos, 0 apoyados por sus Profesores de grupo, la forma de entender los contenidos de las materias, así como la consolidación de sus aprendizajes, pasando desapercibidos por aquellos estudiosos del área; más aún, su esfuerzo es de tomarse en cuenta, así como los Enfoques de Aprendizaje que emplean y el nivel de uso, sobre todo en materias como la de Español, que se encuentra ligada en extremo a sus dificultades, situaciones poco difundidas, o al menos no localizadas por nuestro equipo, para dar cuenta de una robusta cantidad de hallazgos realizados por otros investigadores tanto en América latina, como en México, menos aún en el estado de Chihuahua, afirmación que concuerda con lo mencionado en España por Barca (2002), sobre la pertinencia de explorar los Enfoques de Aprendizaje en poblaciones con dificultades de aprendizaje; por otra parte, la alta representatividad de la muestra aumenta el grado de relevancia a los hallazgos de este estudio.

La conformación de la población de estudio es acorde con lo referido por Málaga y Arias (2010) y el APA en el (2014) donde los varones que cursan con Trastornos de Aprendizaje son una población más grande que la de las niñas; asimismo, las niñas obtuvieron mejores promedios que ellos, reflejando que el grado de deficiencias es mayor en los niños que en ellas, como muestran los resultados. En cuanto a la edad, resalta que existe un porcentaje mayoritario de Alumnos de 11 años; esto refleja con claridad las políticas educativas actuales de México, acorde con la teoría constructivista que las rige, donde la edad va en relación dialéctica entre la experiencia educativa con el grado de madurez que el niño alcanza desde los dos años obligatorios del preescolar, incursando por lo tanto en la Primaria a los 5 y 6 años, llegando al $6^{\circ}$ grado entre los 10 y 12 años, con un sesgo notorio en los 11 , aun cuando sus dificultades de aprendizaje no han sido superadas.

Un porcentaje alto de esta población utiliza el Enfoque Profundo desde nivel Moderado, siendo los más (51\%), hasta Continuo, reflejado en aspectos como: el Alumno repasa un tema, tantas veces como le sea necesario, hasta que lo entiende; se siente contento cuando estudia, investiga extramuros sobre el tema que le gusta más allá de lo que le aporta el Profesor, ahonda sobre el tema preguntando al Docente a fin de que le brinde mayor información, se muestra interesado en nuevos temas, estudiar los temas escolares le resulta tan grato como jugar, le dedica tiempo y trabajo constante a estudiar a fin de saber más sobre los temas que le parecen interesantes, lee más lecturas de las que el Docente indica para conocer más sobre el tema. El hallazgo resulta interesante, ya que estos Alumnos presentan limitantes en habilidades académicas como la lectura y la escritura (APA, 2014; Da Fonseca, 2001; Smith et ál., 2004; y Woolfolk, 2010), de tal manera que, la población de estudio al emplear el Enfoque Profundo, refiere que tratan de logran la compresión de lo aprendido en la materia de Español y darle sentido emocional, de valor e importancia como desde tiempo atrás lo habían reportado Barca, Peralbo, Brenlla, Porto y Santamaría, (2005) utilizando sus recursos cognitivos y aprendizajes obtenidos a lo largo de la educación Primaria.

El Enfoque Superficial se encuentra empleado por más de la media de la población (65\%) en los niveles más bajos, como Nulo y Poco; aunque en menor promedio, el 35\% muestra niveles de Moderado a Alto, las particularidades de este enfoque son: el Alumno no presenta interés en estudiar mucho, ni ahondar en los temas extraclase, ni extramuros, pero si desea pasar 
Vega-Villanueva et al - Enfoques de aprendizaje en la materia de español en niños con trastornos de aprendizaje

los exámenes, estudia solo lo que le aporta el Profesor en la escuela y no considera que es necesario hacer mayores esfuerzos, no le gusta la escuela, memoriza para pasar los exámenes solamente, sin estar interesado en profundizar en el tema; es decir, estos Alumnos al emplear el Enfoque Superficial dan cuenta que, estudian la materia con la intención de librarse de las tareas, realizando actividades de bajo nivel cognitivo en el aprendizaje de los contenidos, no para la comprensión sino solo pasar las evaluaciones correspondientes (Biggs, 2010). Este resultado también puede estar asociado a las características propias de los niños con Trastorno de Aprendizaje como son, las alteraciones relacionadas al proceso de atención, la percepción, que a su vez conlleva consecuencias en el procesamiento de la información y el almacenamiento de la memoria, así como las repercusiones de estas alteraciones conllevan a comprometer el proceso de la lecto-escritura y la compresión lectora como lo sostiene Da Fonseca, (2013). Sin embargo, cabe resaltar, que aún con sus propias características, estos niños han logrado avanzar en la educación Primaria en muchas ocasiones sin el apoyo del equipo USAER, ya que la Educación Básica en México no cuenta con la suficiente cobertura para la atención de los niños con necesidades educativas especiales (INEE, 2019). Lo que nos llevaría a considerar el contexto educativo y el diseño curricular de la educación Primaria en México, dado que como lo menciona Biggs $(1987,2010)$, la predominancia de un Enfoque de Aprendizaje estará determinada tanto por los factores propios del Estudiante como el contexto de enseñanza, es decir, los criterios, el diseño, la enseñanza y las evaluaciones de los aprendizajes que la propia Institución educativa determine para sus Estudiantes.

Al comparar el empleo de ambos enfoques, se observó que los Alumnos emplean en forma dominante el Enfoque Profundo, sobre el Enfoque Superficial; estos resultados hacen denotar que tanto en los Estudiantes con Trastornos de Aprendizaje, población de estudio, como los Alumnos sin ningún tipo de necesidad educativa especial muestran esta dominancia, ya que investigaciones previas realizadas en otros contextos encontraron los mismos resultados como las de Maquilón y Hernández (2011) y Maquilón et ál., (2016) en población Española o García (2016) en diferente población mexicana.

Por otra parte, en los resultados se observó que las niñas emplean en mayor medida el Enfoque Profundo que los varones, coincidiendo en su totalidad con los hallazgos de Maquilón y Hernández (2011); Maquilón et ál., (2016) y contrario a lo descubierto por Montealegre, Núñez y Salgado en el (2014), por Salas (1998) en Estudiantes mujeres universitarias en la que predominó el Enfoque Superficial; posteriormente Salas, Santos y Parra (2004) quienes encontraron en otra población universitaria una asociación positiva entre las buenas calificaciones y el Enfoque Profundo en las mujeres; es interesante resaltar esta concordancia con el estudio de Salas, dada la diferencia de ambas poblaciones, considerando el momento del desarrollo de los Alumnos de $6^{\circ}$ grado de Primaria, ya que se encuentran en el umbral de la adolescencia los niños, y en plena fase las niñas, en contraparte de la juventud de los Estudiantes universitarios, cuyos esfuerzos están claramente encaminados a lograr una meta de su vida futura referidas por Craig y Baucum (2009). Como parte implícita del Enfoque Profundo se encuentra la motivación como elemento emocional, que en las niñas se encuentra en mayor medida que en los niños, como se observó en la población de estudio, la iniciativa y el valor hacia el aprendizaje de la materia de Español referido por Mirete, Soro y Maquilón (2015) y contrario a lo esperado en relación a las conductas socioemocionales de la población con Trastornos Aprendizaje, antes descritas por Smith et ál., en el (2004) y posteriormente por Craig y Baucum, en el (2009), quienes han sido referentes muy importantes para estudios en tiempos pasados y aun en el presente respecto a los infantes que cursan con dificultades en el aprendizaje.

En lo que respecta a la relación entre los Enfoques de Aprendizaje y el promedio en la materia de Español de los niños de la ciudad de Chihuahua con Trastornos de Aprendizaje de $6^{\circ}$ grado de Primaria, los resultados no arrojaron relaciones importantes que reportar, pues si bien se observó que existe una relación negativa y significativa entre Calificación y el Enfoque Superficial, ésta tiene un peso tan pequeño que no es posible tomarlo en consideración, siendo contrarios a los 
datos reportado por Barca, Regina, Brenlla y Santamaría (2000a, 2000b); Barca et ál. (2005); Mirete, et. ál. (2015); Soto, García-Señorán y González (2012) y De la Fuente, Martínez-Vicente, Salmerón, Vera y Cardelle-Elawar (2016) quienes observaron una asociación entre el Enfoque Profundo y mayor rendimiento académico; no obstante, hay que resaltar que la población estudiada por los investigadores citados eran Alumnos Regulares, es decir, sin deficiencias en el aprendizaje como la población del presente trabajo de investigación.

\section{CONCLUSIONES}

Los Estudiantes con Trastornos de Aprendizaje que cursan $6^{\circ}$ grado de Primaria, en escuelas públicas de la ciudad de Chihuahua, Chih., emplean tanto el Enfoque Profundo como el Enfoque Superficial al estudiar para lograr los objetivos marcados en la materia de Español en nivel distinto de frecuencia. Dos terceras partes de la población emplean el Enfoque Profundo en nivel Moderado, Alto y Continuo, y en el Enfoque Superficial solo la tercera parte de la población se localiza en los niveles Moderado o Alto, no llegando al nivel Continuo.

Si bien, los Alumnos con Trastornos de Aprendizaje de $6^{\circ}$ grado emplean ambos enfoques para estudiar la materia de Español, el Enfoque Profundo predomina sobre el Enfoque Superficial, negando la Hipótesis Nula Uno con este hallazgo.

No se encontró relación entre las calificaciones anuales de la materia de Español y el empleo de los Enfoques de Aprendizaje en los niños con Trastornos de Aprendizaje de $6^{\circ}$ Grado de Primaria; ante estos resultados no se puede negar la Hipótesis Nula Dos.

Se afirma que las niñas con Trastornos de Aprendizaje entre 10 y 12 años emplean, en mayor medida que los niños, el Enfoque Profundo al estudiar la materia de Español en $6^{\circ}$ Grado de Primaria, negando la Hipótesis Nula Tres con estos resultados. Por otra parte, los niños con Trastornos de Aprendizaje emplean en mayor medida que las niñas el Enfoque Superficial para estudiar la materia de Español y así poder continuar con el siguiente grado escolar.

Por su gran tamaño, la muestra de esta población da cuenta del empleo, medida, dominancia y predominancia por género de los Enfoques de Aprendizaje de los niños de 10, 11 y 12 años de edad cronológica de la ciudad de Chihuahua, Chih, México, que cursan con algún tipo de Trastorno de Aprendizaje no grave, que se encuentren estudiando nivel escolar de Primaria, y por la representación que tienen la población de niños de esta ciudad, es posible deducir que estos resultados dan cuenta del empleo de los Enfoques de Aprendizaje de los niños con estas características en todo el estado de Chihuahua, más aún se enfatiza que Chihuahua es el estado más grande de la República Mexicana.

Limitantes. Las propias de traslado por las distancias entre una escuela y otra dada la gran extensión geográfica de la ciudad de Chihuahua y la distribución de las escuelas en la misma, así como las asociadas a las características específicas de la población de estudio, a quienes hubo que mantener atentos a la aplicación del instrumento a base del manejo del volumen de la voz por parte del equipo de investigación, así como la imposibilidad de acceder a las Instituciones escolares en algunas ocasiones por eventos de violencia social producidos por el crimen organizado en la ciudad, obstaculizando la planeación exacta del proyecto, más no impidiendo que se llevara a cabo.

\section{Conflicto de intereses}

Los autores declaran no tener ningún conflicto de intereses. 
Vega-Villanueva et al - Enfoques de aprendizaje en la materia de español en niños con trastornos de aprendizaje

\section{Agradecimientos}

Por parte de la Investigadora responsable, al Consejo Nacional de Ciencia y Tecnología (CONACYT), Becaria en el Doctorado en Educación, Artes y Humanidades de la Universidad Autónoma de Chihuahua; a los Servicios Educativos del Estado de Chihuahua, en especial a la Secretaría Académica, por el apoyo en la gestión con las Escuelas Primarias para recabar los datos, a todos los padres que permitieron la aplicación del instrumento a sus hijos y especialmente a los Alumnos por su alta disposición a participar en esta investigación.

\section{REFERENCIAS}

Aguerrondo, I., y Vaillant, D. (2015). Aprendizaje bajo la lupa: Nuevas perspectivas para América Latina y el Caribe. UNICEF. https://www.unicef.org/lac/UNICEF Aprendizaje bajo la lupa nov2015(1).pdf

American Psychiatric Association (2014). DSM-5 Manual diagnóstico y estadístico de los trastornos mentales. 5 a Edición Texto Revisado. Medicapanamericana.

Barca, A. (2002). La escala SIACEPA: Un sistema integrado de evaluación de atribuciones causales y enfoques de aprendizaje para Alumnos de educación secundaria. En J. Escoriza y A. Barca (coord.). Dificultades de aprendizaje: Contenidos teóricos y actividades prácticas. (pp. 139154). Ediciones de la Universidad de Barcelona.

Barca, A., Peralbo, M., Brenlla, J., Porto, A., y Santamaría, S. (2005). Enfoques de aprendizaje, rendimiento académico y usos lingüísticos (castellaño/parlante vs bilingües) en Alumnos de secundaria obligatoria: Un análisis diferencial. Papeles Salmantinos de Educación, 4..14- 34 https://dialnet.unirioja.es/servlet/articulo?codigo=2006755

Barca, A., Regina, C., Brenlla, J., y Santamaría, S. (2000a). Enfoques de aprendizaje, estilos atribucionales y rendimiento académico en una muestra de Alumnos de educación de Brasil. Revista Galego-Portugués de Psicopedagoxía e Educación: Revista de Estudios e Investigación en Psicología y Educación, 6(4), 769 -791. https://dialnet.unirioja.es/servlet/ articulo?codigo $=1049258$

Barca, A., Regina, C., Brenlla, J.y Santamaría, S. (2000b). Propiedades psicométricas de la escala SIACEPA (Sistema integrado de evaluación de atribuciones causales y procesos de aprendizaje) en una muestra de Alumnos de educación secundaria de Brasil. Revista GalegoPortugués de Psicopedagoxía e Educación: Revista de Estudios e Investigación en Psicología y Educación, 6(4), 793-815. https://dialnet.unirioja.es/servlet/articulo?codigo=1049333

Biggs, J, Kember, D \& Leung D. (2001). The revised two - factor study process questionnaire R-SPQ-2F. British Journal of Educational Psychology, 71, 133 - 149. https://doi. org/10.1348/000709901158433

Biggs, J. (2010). Calidad del aprendizaje universitario. (Trad. P. Manzano). Asociación Nacional de Universidades e Instituciones de Educación Superior, Dirección de Medios Editoriales: Narcea

Calderón, R. (1998). El niño con disfunción cerebral. Limusa.

Craig, G., y Baucum, D. (2009). Desarrollo psicológico. Pearson

Da Fonseca, V. (2013). Dificultades de aprendizaje. Trillas. 
De la Fuente, J.; Martínez - Vicente, J.; Salmerón, J.; Vera, M. y Cardelle - Elawar, M. (2016). Estilo acción-emoción, enfoque de aprendizaje y estrategias de afrontamiento, en Estudiantes universitarios de preGrado. Anales De Psicología / Annals of Psychology , 32(2), 457- 465. https://doi.org/10.6018/analesps.32.2.197991

Entwistle, N., y Ramsden, P. (1983). Understanding student learning. Social Science Research Council. http://files.eric.ed.gov/fulltext/ED244959.pdf

García, C. (2016). Estudio comparativo de los enfoques de aprendizaje en Alumnos de educación básica. Recuperado de: http://www.universidadabierta.edu.mx/ActaEducativa/articulos/63. pdf

García. J. y González D. (2001). Dificultades de aprendizaje e intervención psicopedagógica. Fundamentos Psicopedagógicos.

Gearheart. B. (1987). Incapacidad para el aprendizaje. Manual Moderno

Instituto Nacional de Estadística y Geografía. (2010a). Censo de población y vivienda 2010: Tabulados del cuestionario ampliado. Reporte sobre Población total por tamaño de localidad y sexo según condición y tipo de limitación en la actividad. INEGI. http://www.beta.inegi. org. $\mathrm{mx} /$ contenidos/proyectos/ccpv/2010/tabulados/Ampliado/06 06A_ESTATAL.pdf

Instituto Nacional de Estadística y Geografía. (2010b). Censo de Población y Vivienda 2010: Tabulados del Cuestionario Básico. Reporte sobre Población total por municipio, sexo y grupos quinquenales de edad según condición y tipo de limitación en la actividad. INEGI. http://www.beta.inegi.org.mx/contenidos/proyectos/ccpv/2010/tabulados/Basico/06 02B MUNICIPAL 08.pdf

Instituto Nacional para la Evaluación Educativa. (2019). La educación obligatoria en México. Informe 2019. INEE. https://www.inee.edu.mx/wp-content/uploads/2019/04/P1I245.pdf

Málaga, I y Arias, J. (2010). Los Trastornos de Aprendizaje. Definición de los distintos tipos y sus bases neurobiológicas. Boletín de la Sociedad de Pediatría de Asturias, Cantabria, Castilla y Leon, 50, 43-47. https://www.sccalp.org/documents/0000/1526/BolPediatr2010 50 043-047.pdf

Marton, F. y Säljö, R. (2005). Approaches to learning. En The experience of learning: Implication for teaching study of higher education (3a ed.) (Internet) (pp. 39- 58). The University of Edinburgh. Center for teaching. http://www.docs.hss.ed.ac.uk/iad/Learning teaching/ Academic teaching/Resources/Experience of learning/EoLChapter3.pdf

Maquilón, J., y Hernández, F. (2011). Identificación de las características del aprendizaje de los Estudiantes de educación Primaria con el cuestionario CEAPS. Revista Anales de Psicología, 27(1), 126-134. https://dialnet.unirioja.es/servlet/articulo?codigo=3353530

Maquilón, J., Sanchéz, M., y Cuestas, J. (2016). Enseñar a aprender en las aulas de educación Primaria. Revista Electrónica de Investigación, 18(2), 144-155. https://redie.uabc.mx/redie/ article/view/955

Mirete, A., Soro, M. y Maquilón, J.(2015). El fracaso escolar y los enfoques de aprendizaje: Medida para la incluisión educativa. Revista Electrónica Interuniversitaria de Formación del Profesorado, 18(3), 183-196. http://www.redalyc.org/pdf/2170/217042307015.pdf

Montealegre, G., Nuñéz M.y Salgado, D. (2014). Enfoques de aprendizaje y variables de orden sociocultural en estudantes de una Institución de educación superior en Colombia. Acta Medica Colombiana, 39 (4), 368- 377. http://www.scielo.org.co/pdf/amc/v39n4/v39n4a10. pdf 
Vega-Villanueva et al - Enfoques de aprendizaje en la materia de español en niños con trastornos de aprendizaje

Organización para la Cooperación y el Desarrollo Económico. (2019). Programa para la evaluación internacional de Alumnos (PISA) 2018- Resultados. México. https://www. compareyourcountry.org/pisa/country/MEX?lg=en

Organización para la Cooperación y el Desarrollo Económico, Organización de la Naciones Unidas para la Educación, la Ciencia y la Cultura, \& Fondo de las Naciones Unidas para la Infancia. (2016). La naturareza del aprendizaje: Usando la investigación para inspirar la práctica.. https://www.unicef.org/lac/20160505 UNICEF UNESCO OECD Naturaleza Aprendizaje. pdf

Organización de las Naciones Unidas para la Educación, la Ciencia y la Cultura. (2016). Informe de resultados tercer estudio regional comparativo y explicativo. Logros de aprendizaje. Julio 2015. http://unesdoc.unesco.org/images/0024/002435/243532S.pdf

Salas, R. (1998). Enfoques de Aprendizaje entre Estudiantes universitarios. Estudios Pedagógicos, 24, 59-78. https://www.redalyc.org/articulo.oa?id $=173513846005$

Salas. R.; Santos. M.y Parra, S. (2004). Enfoques de aprendizaje y dominancias cerebrales entre Estudiantes universitarios. Aula Abierta, 84, 3-22. https://www.researchgate.net/ publication/39220515 Enfoques de aprendizaje y dominancias cerebrales entre Estudiantes universita

Sánchez, N. (2010). Memoria y actualidad en la Educación Especial en México: Una visión histórica de sus modelos de atención. México, SEP. http://educacionespecial.sepdf.gob.mx/historia/ docs/HistEduEspWeb.pdf

Secretaría de Educación Pública (2013). Programa Sectorial de Educación 2013-2018.SEP. http:// www.sep.gob.mx/work/models/sep1/Resource/4479/4/images/PROGRAMA SECTORIAL DE EDUCACION 20132018 WEB.pdf

Secretaría de Educación Pública (2017a). Modelo Educativo. México. SEP. ISBN:978-607-969033-5. https://www.gob. $\mathrm{mx} / \mathrm{cms} / \mathrm{uploads} /$ attachment/file/198738/Modelo Educativo para la Educacio $n$ Obligatoria.pdf

Servicios Educativos del Estado de Chihuahua (2019). Estadística para PBR 2019. http://seech. gob.mx/estadistica/2019-documentos.asp

Soto, J.; García-Señorán, M y González, S. (2012). Enfoques y estrategias de aprendizaje: un binomio para comprender el rendimiento en la educación secundaria. Revista de Investigación Educativa (10) 2, 95-108. https://dialnet.unirioja.es/servlet/articulo?codigo $=4733215$

Smith, T, Polloway, E., Patton, J y Dowdy, C. (2004). Enseñanza a Estudiantes con necesidades especiales en ambientes incluyentes. Pro-Ed Latinoamérica.

Soler, M. (2014). El constructo Enfoques de Aprendizaje: Un análisis bibliométrico de las publicaciones en Español en los últimos 20 años. Revista Colombiana de Educación, 66, 129-150. http://www.scielo.org.co/pdf/rcde/n66/n66a06.pdf

Soler, M., Cárdenas, F., Hernández, F., y Monroy, F. (2017). Enfoques de aprendizaje y enfoques de enseñanza: Orígenes y evolución. Educación y Educadores, 20(1), 65-88. http: //doi. org/10.5294/edu.2017.20.1.4

Woolfolk. A.(2010). Psicología educativa. Pearson. 